\title{
Processing and Electrochemical Property Characterization of Nanoporous Electrodes for Sustainable Energy Applications
}

\author{
Jenny X. He, ${ }^{1}$ Shruti Baharani, ${ }^{2}$ and Yong X. Gan ${ }^{1,2}$ \\ ${ }^{1}$ Department of Mechanical Engineering, Albert Nerken School of Engineering, \\ The Cooper Union for the Advancement of Science and Art, New York, NY 10003, USA \\ ${ }^{2}$ Department of Mechanical, Industrial and Manufacturing Engineering, \\ College of Engineering, University of Toledo, Toledo, $\mathrm{OH} 43606$, USA \\ Correspondence should be addressed to Yong X. Gan, yong.gan@utoledo.edu \\ Received 20 May 2009; Accepted 23 June 2009 \\ Recommended by Chuan-Jian Zhong
}

Preparation and electrocatalytic reactions of nanoporous materials in biodegradable fluids were studied. Electrochemical etching was conducted to selectively extract metallic elements from alloys to form porous structures. Electrocatalytic properties of the porous electrodes were characterized. Comparative studies on the electrochemical activities of the nanoporous metallic electrodes with bulk metallic wire catalysts were performed. It is found that the current density at the nanoporous electrode is three times higher than that of the bulk electrode.

Copyright (C) 2009 Jenny X. He et al. This is an open access article distributed under the Creative Commons Attribution License, which permits unrestricted use, distribution, and reproduction in any medium, provided the original work is properly cited.

\section{Introduction}

Nanoporous materials may be made via various methods. Chemical etching is an easy way to make nanoporous metals [1-3], while co-evaporation may be used for preparation of nanoporous metal oxides [4]. Nanoporous Au specimens with ligament-like structures were fabricated by dealloying [1]. Smith et al. [2] prepared nanoporous gold films through de-alloying $\mathrm{AuAl}_{2}$. The nanoporous gold has void densities between $45 \%$ and $65 \%$. Zhou et al. [3] proposed another way to fabricate nanoporous gold film. The starting materials are copper and gold alloys thin films obtained through vacuum deposition. The formation of ultra-thin nanoporous gold films from the Au-Cu alloy films involved chemical etching by hydrochloric acid or by nitric acid.

It is well known that preparing nanoporous metals via chemical etching is very difficult to control in view of the reaction kinetics. For example, the change in the concentration of the acids, and the temperature fluctuation in the etchant solutions have significant effect on the ligament morphology, void density and strength of the nanoporous metals. During the process of pore formation, the capillary effect causes surface relaxation and affects the dimension of the nanoporous metals [5]. Another drawback of chemical dealloying is the environmental pollution due to the frequent use of high concentration, volatile enchants such as concentrated hydrochloric acid and/or nitric acid. One of the objectives of this work is to develop a better controllable dealloying process than chemical etching. Preliminary research results of electrochemical dealloying in low concentration acids to generate nanoporous nickel and gold will be presented. Electrocatalytic properties of the nanoporous $\mathrm{Ni}$ and $\mathrm{Au}$ in agricultural products for sustainable energy applications will be evaluated.

\section{Materials and Experiments}

Annealed gold-nickel wire with the composition of $\mathrm{Au}: \mathrm{Ni}=$ $82: 18$ wt $\%$ was purchased from Alfa Aesar, Ward Hill, $\mathrm{Md}$, USA. The diameter of the Au-Ni wire is $0.5 \mathrm{~mm}$. Copper-nickel wire with the diameter of $0.5 \mathrm{~mm}$ was also purchased from the same company. The composition of the $\mathrm{Cu}-\mathrm{Ni}$ wire is $\mathrm{Cu}: \mathrm{Ni}=55: 45 \mathrm{wt} \%$. Platinum with the diameter of $0.25 \mathrm{~mm}$ and the purity of $99.997 \mathrm{wt} \%$ was purchased from Alfa Aesar. Other chemicals such as hydrochloride acid, sulfuric acid, acetone with ACS purities were ordered from Alfa Aesar. Four biodegradable materials 


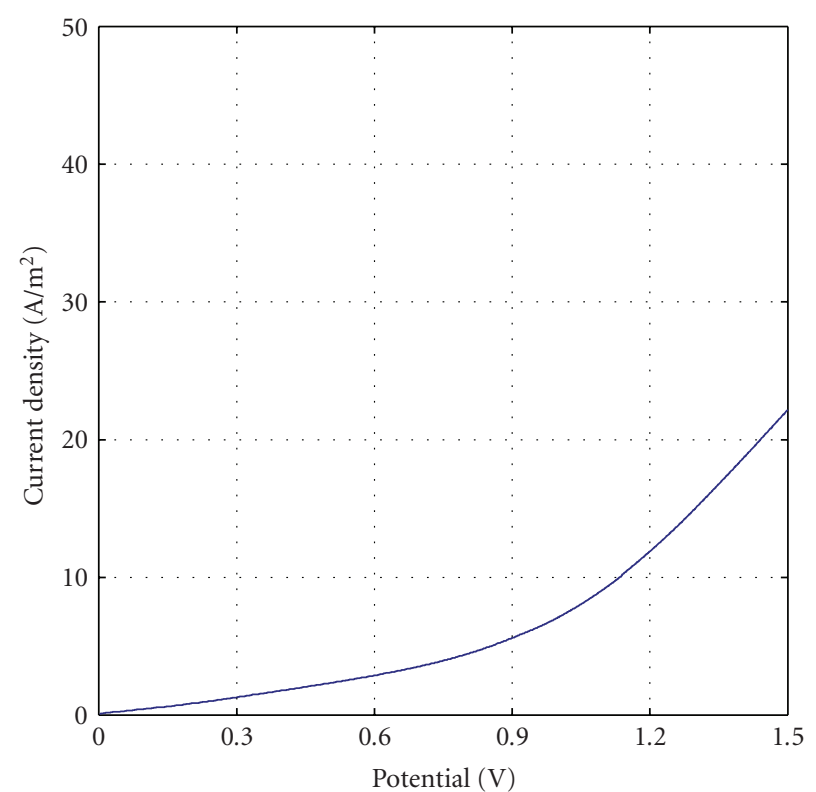

(a)

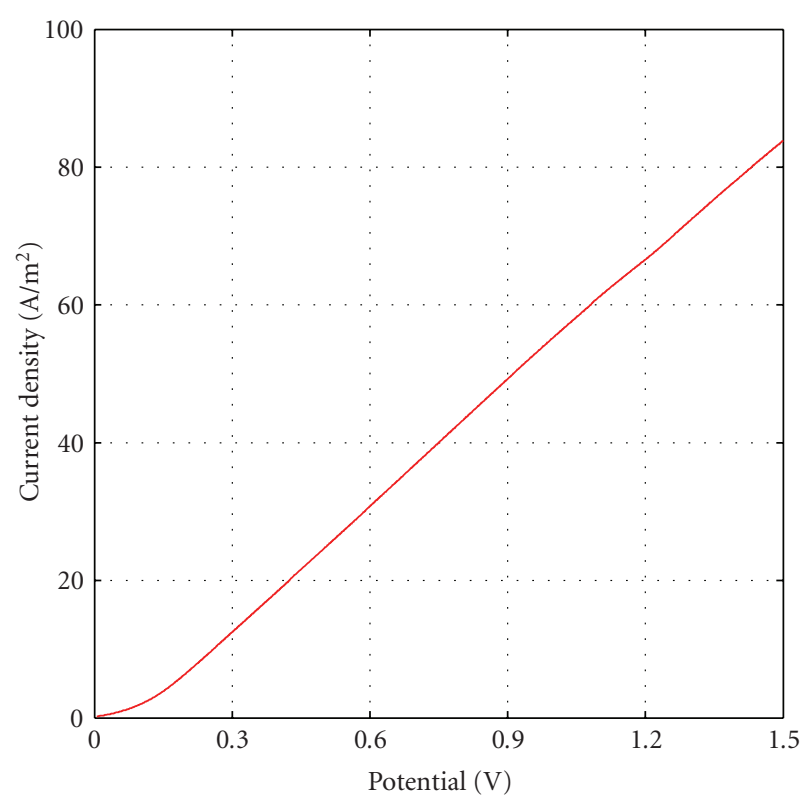

(b)

FIGURE 1: Cyclic voltammograms of Ni electrode in grapefruit juice: (a) without pore; (b) with pores.

were used in characterizing the electrocatalytic behaviors of the nanoporous $\mathrm{Au}$ and Ni. They are tomato, milk, chocolate drink and grape fruit juice. All these products were purchased from Trade Fair, Forest Hills, USA. A three-electrode system was set up for both electrochemical dealloying and electrocatalytic property characterization. The electrodes are connected with a CHI600B Electrochemical Analyzer. The three electrodes are the $\mathrm{Ag} / \mathrm{AgCl}$ reference electrode, the work electrode, and the platinum counter electrode. Selective removal of $\mathrm{Ni}$ for $\mathrm{Au}-\mathrm{Ni}$ alloy and $\mathrm{Cu}$ from $\mathrm{Cu}-$ $\mathrm{Ni}$ alloy by electrochemical etching in diluted $\mathrm{HCl}$ and $\mathrm{H}_{2} \mathrm{SO}_{4}$ was conducted. First, $\mathrm{HCl}$ aqueous solution with the concentration of $9 \mathrm{wt} \%$ was made. The $\mathrm{H}_{2} \mathrm{SO}_{4}$ used has the concentration of $25 \mathrm{wt} \%$. Second, the $\mathrm{Au}-\mathrm{Ni}$ and $\mathrm{Cu}-\mathrm{Ni}$ were made into two separate work electrodes. Each work electrode was assembled with the $\mathrm{Ag} / \mathrm{AgCl}$ reference electrode, and the platinum counter electrode in the cell containing $\mathrm{HCl}$ or $\mathrm{H}_{2} \mathrm{SO}_{4}$ solution. Under controlled potentials generated by positive cyclic voltammetrical (CV) scan, selective dissolving of metal elements was achieved. As a result, nanoporous $\mathrm{Au}$ and nanoporous Ni were obtained. The electrocatalytic activities of the nanoporous $\mathrm{Au}$ and nanoporous $\mathrm{Ni}$ in the biodegradable substances of fresh tomato, milk, chocolate drink, and grape fruit juice, respectively, were characterized by cyclic voltammetry $(\mathrm{CV})$. The scanning rate is $0.01 \mathrm{~V} / \mathrm{s}$. The tests were done at the same temperature of $25^{\circ}$.

\section{Results and Discussion}

3.1. Effect of Nanopores on Catalytic Oxidation Behaviors. In order to examine the effect of nanopore on the electrocatalytic behavior of electrodes in biodegradable fluids, comparative studies on nanoporous nickel and bulk nickel were conducted. Electrochemical catalysis behavior of both bulk $\mathrm{Ni}$ and nanoporous $\mathrm{Ni}$ in the grape fruit juice was studied by cyclic voltammetry and the results are shown in Figures 1(a) and 1(b). The current density at the nanoporous electrode, as shown in Figure 1(b), is calculated based on the initial area of the alloy before dealloying. The cyclic voltammogram of the bulk $\mathrm{Ni}$ as shown in Figure 1(a) has the reaction controlled feature as the porous Ni electrode. However, the current density at the nanoporous $\mathrm{Ni}$ is as 4 times high as that at the bulk nickel electrode, which reveals that the nanoporous electrode is more effective than the bulk $\mathrm{Ni}$ in electrochemical catalytic oxidation of the grape fruit juice.

Some insights into calculating the area of nanoporous material can be obtained from the results. Typically, the charge associated with the electrochemical catalytic oxidation is proportional to the real surface area of the electrode. Therefore, the magnitude of the current density can be used to calculate the surface area of the electrode. It is shown that the current density for the electrode increased due to the dealloying of copper from the nickel-copper alloy wire. The results in Figure 1 indicates that the real electrochemically active area of the pore electrode is about 3 times higher than the bulk wire material. Similar results were also obtained for gold-nickel material.

The electrocatalytic behavior of the above used $\mathrm{Ni}$ electrodes without nanopores and with nanopores in fresh tomato was studied. At the same potential, the current density at the bulk nickel electrode shown in Figure 2(a) is lower than that at the nanoporous nickel electrode shown in Figure 2(b). This trend is the same as found in the grape fruit juice case. The oxidation of tomato on the bulk electrode 


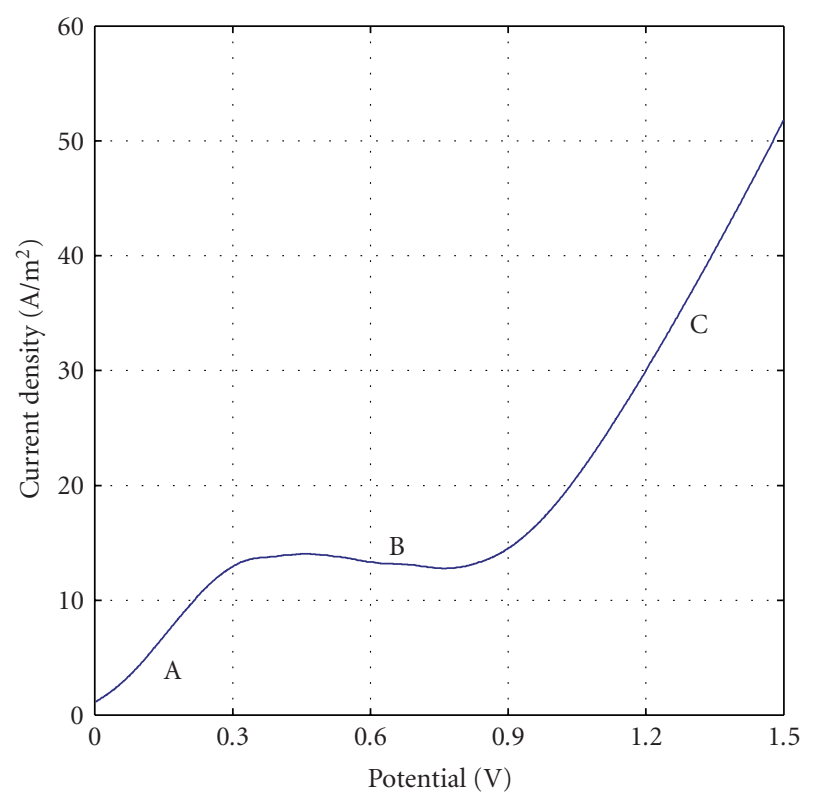

(a)

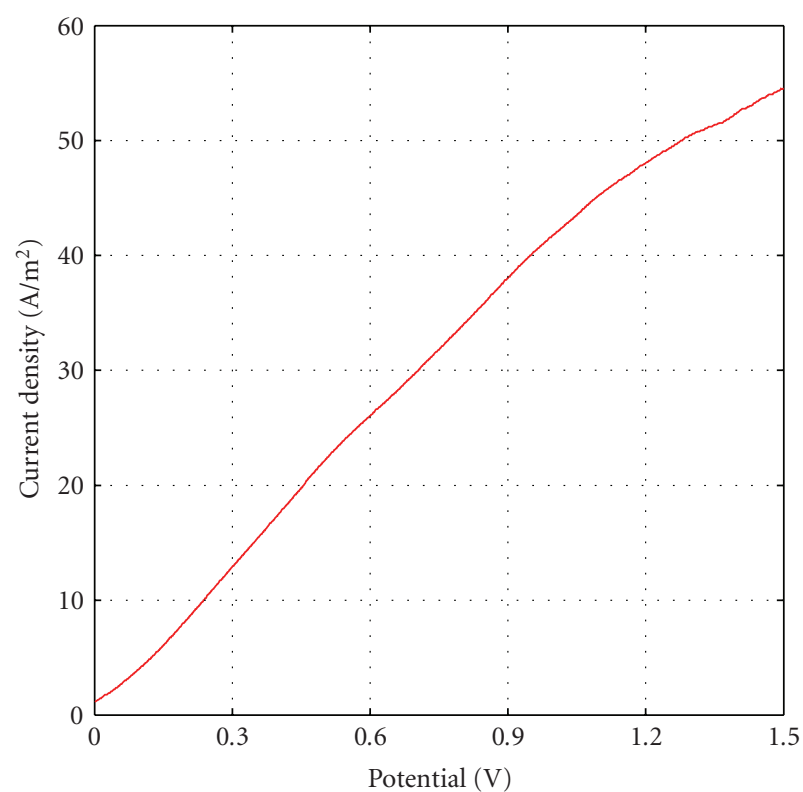

(b)

FIGURE 2: Cyclic voltammograms of Ni electrode in tomato : (a) without pore ; (b) with pores.

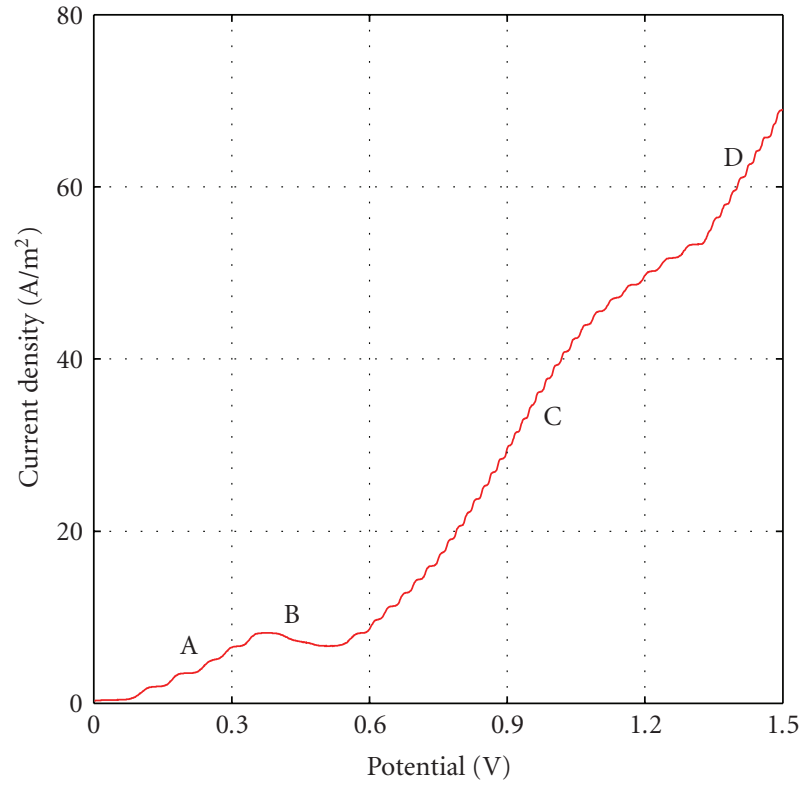

(a)

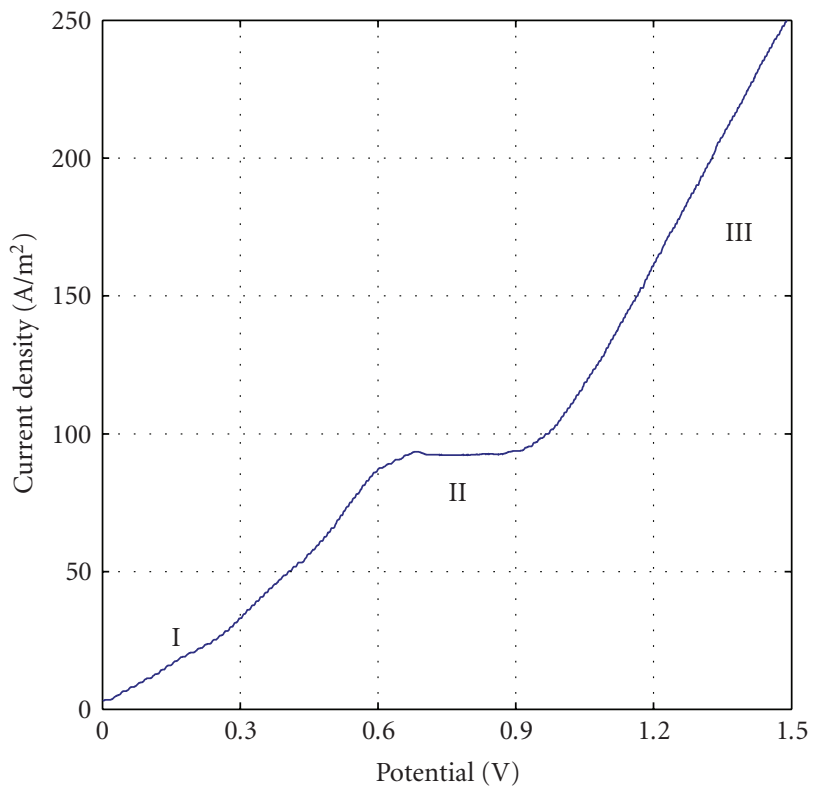

(b)

FIGURE 3: Cyclic voltammograms of the nanoporous $\mathrm{Au}$ and nanoporous Ni in milk: (a) nanoporous Au; (b) nanoporous Ni.

has three distinct stages as mark by "A", "B", and "C" on the cyclic voltammogram of Figure 2(a). In stage " $\mathrm{A}$ " and " $\mathrm{C}$ ", oxidation reactions are the controlled processes. In stage " $\mathrm{B}$ ", the diffusion controlled feature is shown. At the nanoporous $\mathrm{Ni}$ electrode, only the reaction controlled behavior in the tomato juice was found, which is revealed by the cyclic voltammetry results in Figure 2(b).
3.2. Effect of Metal Type on Catalytic Oxidation Behaviors. The electrochemical catalytic behavior of both nanoporous nickel and nanoporous gold in biodegradable fluids was studied to examine the effect of metal types. Both the nanoporous $\mathrm{Au}$ and nanoporous $\mathrm{Ni}$ were prepared by electrochemical dealloying in the $9 \% \mathrm{HCl}$ electrolyte. Figure 3(a), the cyclic voltammogram of nanoporous Au in 


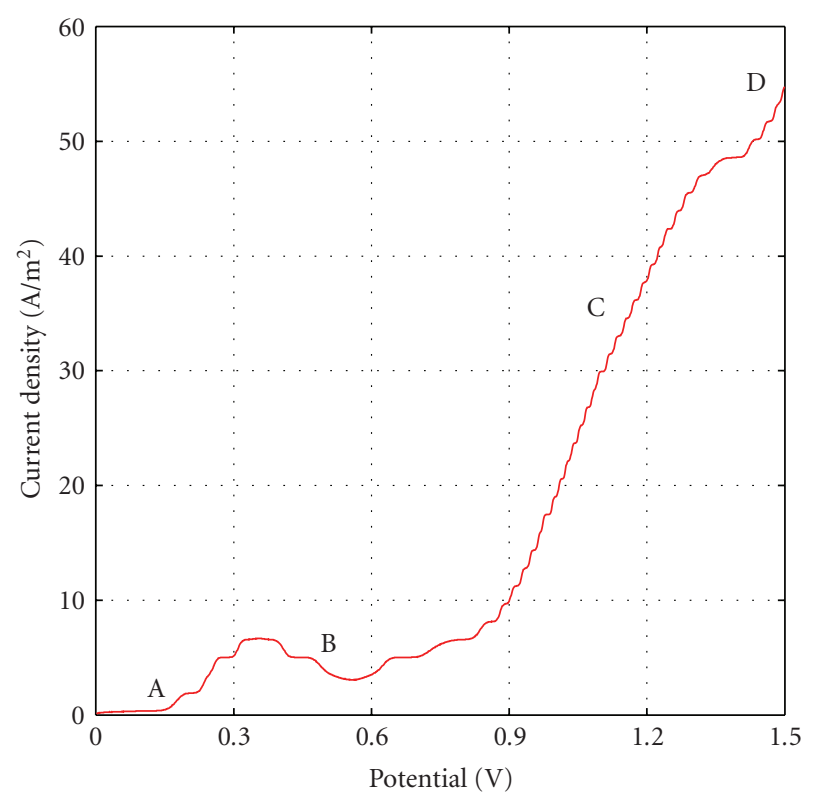

(a)

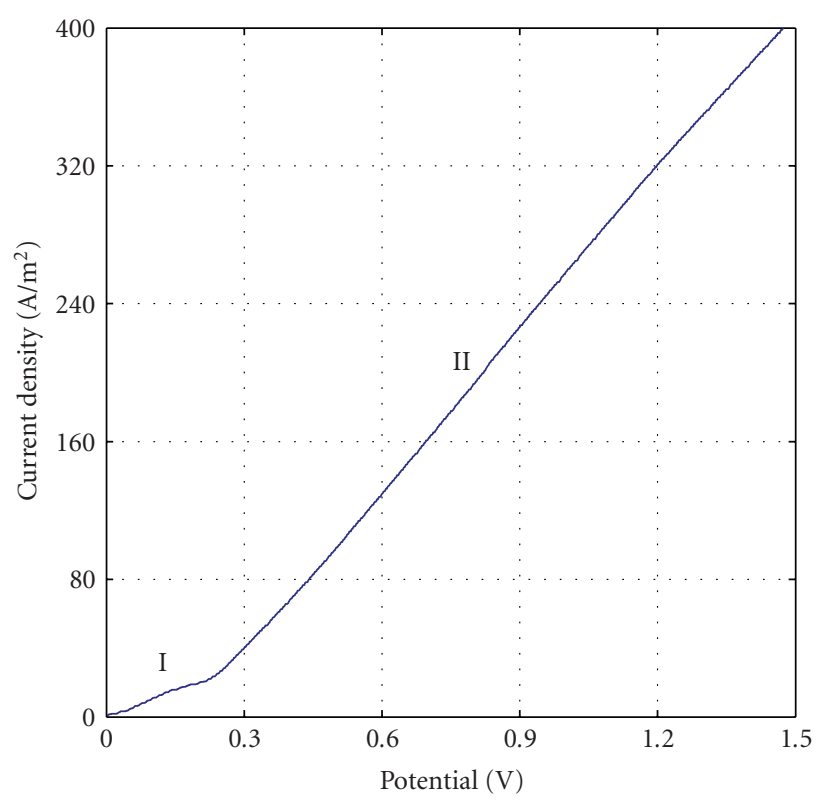

(b)

FIGURE 4: Cyclic voltammograms of the nanoporous Au and nanoporous Ni in chocolate dink: (a)nanoporous Au; (b) nanoporous Ni.

the milk, shows four stages of electrochemical oxidation as marked by "A", "B", "C", and "D". One of the reasons for such a complex cyclic voltammogram is due to the complexity of the composition of the milk. The cyclic voltammogram of nanoporous $\mathrm{Ni}$ in the milk, also shows a multi-stage electrochemical oxidation behavior as marked by "I", "II", and "III" in Figure 3(b). Stages "I" and "III" are reaction controlled behaviors, while stage "II" is the diffusion-controlled behavior. At any given potential, the current density at the nanoporous $\mathrm{Ni}$ electrode is higher than that at the nanoporous Au electrode. This is reasonable because the nominal porosity of the nanoporous $\mathrm{Au}$ is about $18 \%$, while this value for the nanoporous $\mathrm{Ni}$ is about $55 \%$. The nanoporous Ni has much higher surface area than that of the nanoporous $\mathrm{Au}$. Therefore, the specific current calculated based on the initial surface area for the nanoporous $\mathrm{Ni}$ electrode is higher.

The electrochemical catalytic behavior of the nanoporous nickel and nanoporous gold in the chocolate drink was also studied. Figure 4(a), the cyclic voltammogram of nanoporous $\mathrm{Au}$ in the chocolate drink, reveals four stages of electrochemical oxidation as marked by "A", "B", "C", and "D". The cyclic voltammogram obtained from the test in the chocolate drink is very similar to that obtained from the milk. This is because the compositions of the two biodegradable solutions are very close. The cyclic voltammogram of nanoporous $\mathrm{Ni}$ in the chocolate drink only shows two distinct stages denoted by "I", and "II" in Figure 4(b). Both stages "I" and "II" are under reaction control. Similar to the results shown in Figure 3(a) and 3(b), the current density at the nanoporous $\mathrm{Ni}$ electrode is higher than that at the nanoporous Au electrode because both the nominal porosity and the active surface area of the nanoporous $\mathrm{Ni}$ are higher than those of the nanoporous Au.
It should be pointed out that for electrochemical oxidation of many materials, $\mathrm{pH}$ value may have very significant effect on the process. In this paper, we measured the $\mathrm{pH}$ value of the fluids. The $\mathrm{pH}$ value for fresh tomato is around 6.0. The $\mathrm{pH}$ value for chocolate drink is 6.5. The $\mathrm{pH}$ value for the grape fruit juice is 6.3. After the electrochemical tests, the $\mathrm{pH}$ value for each fluid dropped less than 0.1 unit. Such a little change in the $\mathrm{pH}$ value might cause the slightly shift of oxidation peak according the calculation by Nerst equation, but it should not change the characteristic peaks. Therefore it is concluded that the difference in electrocatalytic properties of different fluids is not related to the minor change of the $\mathrm{pH}$ value.

Transmission electron microscopic (TEM) study of the porous materials is helpful in examining the high resolution morphology, showing the dislocation structure around the nanopores, determining the crystallographic orientation of the nanopores. This would be one of our future work on the porous materials.

\section{Conclusions}

Electrochemical dealloying and electrocatalytic behaviors of nanoporous metals in biodegradable fluids were performed. High electroactive surface areas in nanoporous nickel and nanoporous gold were obtained. Based on the preliminary research, the following concluding remarks can be made.

Comparative studies on nanoporous and bulk metallic catalysts show that nanoporous electrodes are more effective than bulk electrodes in electrochemical catalytic oxidation of biodegradable fluids. The cyclic voltammogram of the bulk $\mathrm{Ni}$ has the same reaction controlled feature as the porous $\mathrm{Ni}$ electrode. However, the current density at the nanoporous $\mathrm{Ni}$ is as 4 times high as that at the bulk nickel electrode. 
The electrochemical catalytic properties of the nanoporous nickel and nanoporous gold in biodegradable fluids are different. Although multi-stage catalytic oxidation behaviors are found at both types of electrode, the nanoporous nickel shows a reaction controlled electrochemical catalytic behavior. The nanoporous gold shows both reaction and diffusion controlled mechanisms.

\section{Acknowledgments}

This work is supported by the Durbin Faculty Development Grants at The Cooper Union and the Summer Faculty Research Fellowships at The University of Toledo.

\section{References}

[1] M. Hakamada and M. Mabuchi, "Mechanical strength of nanoporous gold fabricated by dealloying," Scripta Materialia, vol. 56, no. 11, pp. 1003-1006, 2007.

[2] G. B. Smith, A. I. Maaroof, and A. Gentle, "Homogenized Lorentz-Drude optical response in highly nanoporous conducting gold layers produced by de-alloying," Optics Communications, vol. 271, no. 1, pp. 263-268, 2007.

[3] H. Zhou, L. Jin, and W. Xu, "New approach to fabricate nanoporous gold film," Chinese Chemical Letters, vol. 18, no. 3, pp. 365-368, 2007.

[4] T. Tesfamichael, N. Motta, T. Bostrom, and J. M. Bell, "Development of porous metal oxide thin films by co-evaporation," Applied Surface Science, vol. 253, no. 11, pp. 4853-4859, 2007.

[5] D. A. Crowson, D. Farkas, and S. G. Corcoran, "Geometric relaxation of nanoporous metals: the role of surface relaxation," Scripta Materialia, vol. 56, no. 11, pp. 919-922, 2007. 

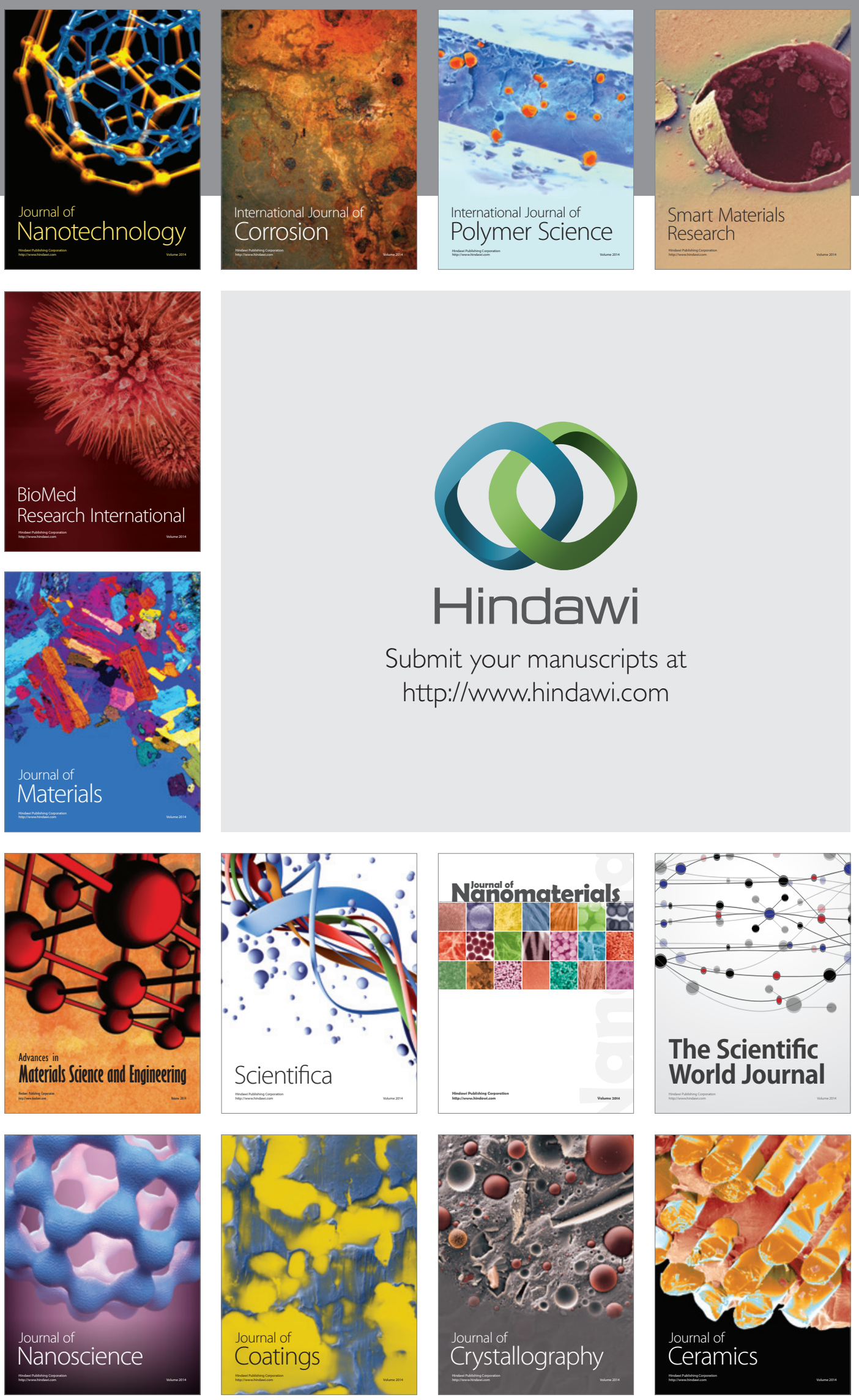

The Scientific World Journal

Submit your manuscripts at

http://www.hindawi.com

\section{World Journal}

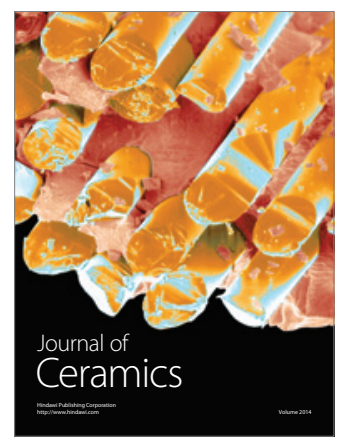

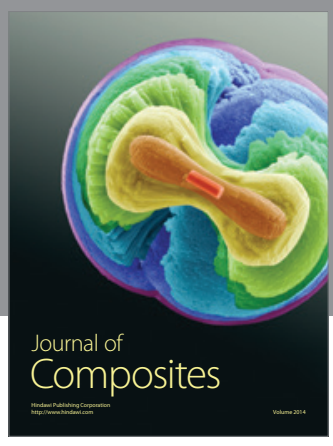
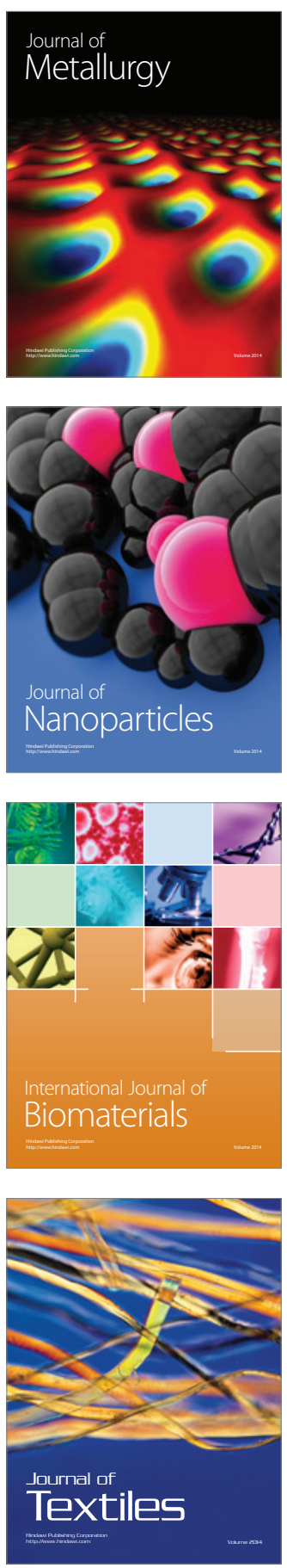